\title{
Host-cue information processing by foraging jewel wasps: Response-bias tracking of expected host encounter rates, not modified profitabilities
}

\author{
AURELIO JOSE FIGUEREDO \\ University of Arizona, Tucson, Arizona
}

\begin{abstract}
Three hundred forty female jewel wasps were reared as larvae on blowfly pupae and then separately exposed as adults to varying numbers and combinations of previously parasitized and unparasitized housefly pupae. Each adult was tested with 6 fresh housefly pupae. Only foraging experience on unparasitized housefly pupae increased host acceptance for subsequent housefly pupae, producing curvilinear and convex host-acceptance functions; foraging experience on preparasitized housefly pupae had no effect upon subsequent host acceptance. Labile hostacceptance thresholds are thus adjusted by foraging experience to track local unparasitized host abundances for different species by modulating host-species cue response biases. These presumably kairomonal response biases were found to be sensitive to psychophysical "subjective probabilities of stimulus presentation," representing expected unparasitized host-species encounter rates, not altered "payoff matrices" of "observer decision outcomes," representing modified hostspecies profitabilities.
\end{abstract}

The purpose of this paper is to investigate the proximate mediation of conditioned host selection in the parasitoid jewel wasp, Nasonia (Mormoniella) vitripennis (Hymenoptera: Pteromalidae), in the light of recent findings (Figueredo, 1989a, 1989b) regarding the effects of both adult foraging and larval feeding experience in that species. It was proposed that our conceptions of the nature of adult host species' "switching," as well as "preimaginal conditioning," be critically reviewed, if not fundamentally revised, with respect to certain complementary perspectives. One was the historically unresolved yet fundamental problem of discriminant validity in behavioral measurement, such as in distinguishing relative changes in host preference from absolute changes in host acceptance. Another was the issue of ultimate causation previously left open by an insufficiently addressed yet required

This research was primarily supported by a National Science Foundation Graduate Fellowship at the University of California, Riverside, and was submitted in partial fulfillment of the requirements for a PhD in psychology. Special thanks are due to Lewis Petrinovich, for guidance in research philosophy, and to Keith Widaman, for training and assistance in the application of the quantitative methodology. Thanks also to Tom Baker, Ken Haynes, Bob Luck, Len Nunney, and Nick Waser in entomology and ecology for their discussion and perspectives on interpretation of these results, and to Alan Bond and Dave Perrott for their ideas on the application of signal detection theory to animal models. The author's research assistants were Russell Bruney, Rob Davis, Valerie Devereaux, Shelia Eiden, Bruce Elkins, Sam Gilbert, Julie Gortsema, Eva Hanloser, Debra Hustad, Mark McCoy, Sandy Messina, Maureen Murphy, Kei Tiggs and Kim Tsutsui. Address correspondence to the author at the Department of Psychology, University of Arizona, Tucson, Arizona 85721. specification of adaptive ecological function, such as may be inferred from a correct description of the operating characteristics of the behavioral mechanism. These and other major issues were comprehensively addressed within the studies cited and are summarized in the Appendix of this paper, prefaced by a brief review of the natural history of the species. However, some more tentative interpretations regarding proximate causation that implicitly generate certain empirically testable predictions were invoked. The experimental confirmation, or disconfirmation, of these theoretical predictions is the subject of the present paper.

Certain results of the studies cited above were difficult to interpret in terms of the unaided theories of optimal foraging. When psychophysical constructs derived from the theory of signal detection (Dember \& Warm, 1979; Kling \& Riggs, 1971) are applied, however, the results strongly suggest plausible mechanisms of proximate mediation for conditioned host selection. These were derived and proposed simply by distinguishing between the perceptual sensitivity to stimuli, or discriminability, and the decision to respond, or response bias, under conditions of perceptual uncertainty. Pursuing the logical consequences of this proposed psychophysical interpretation, the experimental question becomes, Which of the two nonperceptual factors documented in the psychophysical literature, if either, account for the labile response criteria observed? These candidates are the a posteriori "subjective probability of stimulus presentation" (the parasitoid's expected "encounter rate" based upon prior experience) and the expected "payoff matrix" of "observer decision 
outcomes" (the relative "profitabilities" of the alternative host species, as modified by any changes in parasitoid searching or handling times produced by host experience).

Conditioned differences in either the searching or the handling times for either of the two alternative host species were not measured in either of the two preceding studies; therefore, any indirect modifications of relative host-species profitabilities suggested remain no more than hypothetical. It is excessively labor-intensive, however, to directly measure changes in parasitoid searching or handling times for different host species with different parasitoid host-species experience, with the hope of achieving any statistically valid sample size. Furthermore, such observational data would be inconclusive, at best, since they would not necessarily demonstrate that the measured changes in temporal foraging parameters were indeed causal to the host-acceptance threshold effects in question. In the present study, the research strategy was to test the idea of payoff-matrix conditioning by directly manipulating, rather than passively observing, the host-species mean profitabilities, independently of the abundances, experienced during adult foraging.

Although the natural range of variability of gross food value in conspecific host pupae is not great enough to serve as a sufficiently powerful manipulation, the existence of a superior research tool has been reported in the literature. Previously parasitized host pupae are discriminated from unparasitized host pupae by jewel wasps only after the completion of drilling and insertion of the ovipositor, rather than upon superficial contact, such as by the detection of any physical wounds or chemical traces left on the puparium surface by the previous attacker (Edwards, 1954; Whiting, 1967; Wylie, 1958, 1965). The exact identity or combination of cues utilized in discriminating previously parasitized from unparasitized hosts is still uncertain, although it has been suggested that they include an "oviposition-deterring pheromone" in the venom injected into the host, as well as the physical injury inflicted internally by the ovipositor (Wylie, 1965).

Nevertheless, it appears that these cues are unavailable to the parasitoid until all searching, and most of the standard handling time, has been allocated to, and unproductively "invested" in, the preparasitized host candidate. Both the quantity and the quality (in terms of expected fecundity, as well as sex ratio) of the offspring obtainable by superparasitization are substantially reduced, partially by prior ovipositional restraint and partially by scramble competition for host resources by multiple broods during their development (Walker, 1967). This provides a way to manipulate the mean profitability of a sample of hosts without altering either the sizes or the numbers of conspecific fly pupae presented for adult jewel-wasp conditioning.

\section{QUALITATIVE PREDICTIONS}

The most probable outcomes of this study can be roughly categorized into three qualitative classes. (It is a relatively safe prediction that increasing the number of unparasitized hosts presented during conditioning produces a positive effect upon subsequent host acceptance; therefore, any possible effect produced by increasing the number of previously parasitized hosts presented during conditioning becomes the critical outcome.) Exposure to preparasitized hosts during conditioning must produce either a (1) zero, (2) positive, or (3) negative effect upon host acceptance at subsequent testing. These three qualitative alternative outcomes, although mathematically simple to describe, have dramatically divergent behavioral implications.

An outcome of Class 1 would be obtained if presentation of any tested number of preparasitized hosts during conditioning, whether alone or in any combination with unparasitized hosts, produced no significant effect upon subsequent host acceptance. The hypothesis that the hostcue response thresholds in these parasitoids are modulated by expected host-species mean profitabilities would not be supported. This is because, for any given number of (randomly assigned) unparasitized hosts made available, it is the added number of preparasitized hosts also presented that would, on the average, determine the mean profitability of the combined host sample and any gross numerical "practice effect" of host experience that presumably decreased future searching or handling times. The competing hypothesis is that the host-cue response thresholds are modulated by expected gross host-species abundances. This hypothesis would also be somewhat qualified, because only the net abundance of unparasitized hosts would modulate parasitoid responsiveness. This outcome might be comparable to Waage's (1979) simple threshold rate model of optimal patch time allocation for Nemeritis canescens, wherein higher time rates of successful oviposition delay the waning of parasitoid responsiveness to arrestant kairomonal stimuli within a patch of hostinfested habitat. This might mean that only the consummatory response (oviposition) rate, and not the total appetitive stimulus (encounter) rate, is utilized as a hostabundance cue by the insect. A more detailed description of this model is provided in the Appendix of this paper.

Alternatively, an outcome of Class 2 would be obtained if presentation of preparasitized hosts during conditioning, whether alone or in any combination with unparasitized hosts, produced a positive effect upon subsequent host acceptance. The hypothesis that host-cue response thresholds are modulated by expected gross host-species abundances would be more strongly supported; the competing hypothesis that response thresholds are modulated by expected mean host-species profitabilities might also be more strongly disconfirmed if the positive effects of unparasitized and preparasitized hosts were found statistically equivalent. Although such an outcome might also be consistent with a practice effect hypothesis, with gross numerical host experience decreasing future searching or handling times regardless of host quality, it could not be reconciled logically with any proposed parasitoid sensitivity to the future mean host-species profitabilities, be- 
cause two host types significantly differing in average quality would nonetheless have functionally equivalent effects upon subsequent host acceptance. This outcome would instead indicate that the functional, as well as the ecological, validity of every preparasitized host is equal to that of every unparasitized host encountered, as an indicator of expected gross host-species abundance. Whether or not such gross host-species abundance, unweighted by quality, should be of strategic importance to the foraging parasitoid is a matter for optimality theory to address.

Finally, an outcome of Class 3 would be obtained if presentation of preparasitized hosts during conditioning, whether alone or in any combination with unparasitized hosts, produced a negative effect upon subsequent host acceptance. The hypothesis that host-cue response thresholds are modulated by expected host-species mean profitabilities would be strongly supported, particularly so if the effects of unparasitized and preparasitized hosts were found equal and opposite. Such an outcome would contradict both the expected gross host-species abundance and the gross numerical practice effect of host-species experience hypotheses. This outcome would indicate that these parasitoids are tracking host-species expected quality, as well as a "weighted" expected quantity.

\section{Methods}

\section{Subjects}

The parasitoid jewel wasp, Nasonia vitripennis, and two species of dipteran host, Sarcophaga bullata, a carrion blowfly, and Musca domestica, the common housefly, were used in these experiments. Cultures of all three insects were obtained from Carolina Biological Supply Company. Fly pupae were used directly after 1 day of preconditioning in the incubator on a 12:12-h light:dark cycle, at $25^{\circ} \mathrm{C}\left( \pm 2^{\circ} \mathrm{C}\right) 80 \%$ relative humidity ( $\pm 10 \%$ relative humidity). Experimental wasps were obtained by assigning fly pupae of the specified host species to each female wasp for oviposition. The offspring were reared to adulthood in the incubator at the specified photoperiods, temperatures, and humidities; they were fed on honey until the controlled exposures to eliminate any effects of prior feeding experience on pupae other than the larval host. To permit adequate fertilization of all test females, male and female clutchmates were housed together in plastic culture vials until the conditioning or test exposures.

All adult wasps used were blowfly-reared. Previous results (Figueredo, 1987) suggested that their higher fecundity could be expected to minimize any possible problems of nutrient depletion with repeated ovipositions. All conditioning and testing hosts used were housefly pupae, however, since these have shown the greatest variance in acceptance by jewel wasps. Preparasitized hosts were obtained by previously exposing housefly pupae for parasitization to nonexperimental adult female "confederate" jewel wasps, in a standard ratio of 5 to 3 per culture vial, respectively, for a standard preexposure interval of $64 \mathrm{~h}$.

Cohort ages in the specified stage of metamorphosis were as follows, plus or minus 1 day: wasps used as confederates were initially 2 days old as adults, and their housefly hosts were 1 day old as pupae at the start of the preexposure interval; wasps used as experimentals were 6 days old as adults by the start of the conditioning exposure interval, and their housefly hosts were 5 days old as pupae by the start of either the conditioning or the testing intervals, regardless of whether or not they had been previously exposed to the confederates. All exposure-mate conditioning, as well as test- ing, hosts were therefore of the same age as pupae at the time of their successive exposures to the experimentals, regardless of previous exposure to confederates.

For Experiment 1, both the confederate and the experimental wasps were of the homozygous, dominant brown-eyed $(+.+/+.+)$, or wild, genotype; for Experiment 2, the confederates were of the homozygous, recessive scarlet-eyed $(+. s t /+. s t)$ genotype, and the experimentals were of the homozygous, recessive oyster-eyed (oy. +/oy. + ) mutant genotype. Total numbers of insects used were 3,640 housefly pupae and 820 adult wasp females. Of these wasps, 340 were experimentals, of which 180 were brown-eyed wild types and 160 were oyster-eyed mutants, and 480 were confederates, of which 216 were brown-eyed wild types and 264 were scarleteyed mutants.

\section{Procedure}

All of the conditioning and testing exposures in this study were conducted in $32 \times 102-\mathrm{mm}$ plastic culture vials, stoppered with foam plugs, for a standard interval of $24 \mathrm{~h}$. To avoid confounding sibling relatedness with tested choice effects, wasps were systematically assigned from clutches (necessarily within rearing conditions) to different adult conditioning exposures. The adult wasps were refrigerated (at $6^{\circ} \mathrm{C}$ ) for about $6 \mathrm{~min}$ to facilitate handling without chemical anaesthesia. For both conditioning and subsequent testing exposures, one adult female wasp per vial was deposited (using a camel-hair brush) on the inside wall about halfway down to the host pupae that were scattered at the bottom.

The conditioning exposures were conducted with varying numbers and combinations of unparasitized and preparasitized housefly pupae, as specified below. In Experiment 2, all conditioning hosts were afterwards separated into individual test tubes. All wasps were tested immediately after the conditioning exposure with a standard number of 6 unparasitized housefly pupae. Previous results (Figueredo, 1989a) suggested that this number of testing hosts could be used to maximize experimental variance. No between-species preference tests were conducted since single-species conditioning has been shown not to affect host-species preference, regardless of any hypothetical changes in parasitoid subjective expectations of either host abundances or profitabilities.

For Experiment 1, conditioning exposures consisted of the following: (1) an all-unparasitized series of either $0,1,2,3,4,5$, or 6 unparasitized hosts, with 0 preparasitized hosts present, (2) an all-preparasitized series of either $1,2,3,4,5$, or 6 preparasitized hosts, with 0 unparasitized hosts present, and (3) a mixed unparasitized and preparasitized series consisting of a standard total of 6 hosts of which either $1,2,3,4$, or 5 were unparasitized, with the remainder $(5,4,3,2$, and 1 , respectively) of the hosts present being preparasitized. For Experiment 2, conditioning exposures consisted of the following $4 \times 4$ orthogonal matrix: (1) a series of either $0,1,3$, or 7 previously unparasitized pupae, fully "crossed" with (2) a series of either $0,1,3$, or 7 previously parasitized pupae. All 16 combinations of these numbers of previously parasitized and unparasitized host pupae were thereby generated.

At the end of each exposure interval, any ongoing behavior was interrupted and the adult female wasps were removed. Between bouts of handling (such as during any of the timed exposures and subsequent periods for offspring development), all insects were maintained continuously in the incubator under the conditions specified above. Within a few days after each exposure, all surviving test host flies of both species had matured, and within about 2 weeks, most wasp offspring had emerged. Seventeen days after exposure, however, all test hosts that had failed to produce adult flies were dissected for remaining parasitoid adults, pupae, and larvae.

\section{Data Encoding}

A single dependent variable was measured: the number of test hosts successfully parasitized by any given wasp during the testing 
exposure interval, as evidenced by production of living parasitoid progeny at either natural emergence or dissection. The numbers of pupae presented for the adult conditioning of the experimental wasps that had been either preexposed or not preexposed to the confederate wasps, as specified, were symbolized by two independent variables. Of the conditioning hosts presented to each wasp, the absolute number that had not been preexposed, and hence were definitely not preparasitized, was denoted by the discrete, intervalscale variable number of hosts not preexposed. Of the conditioning hosts presented to each wasp, the absolute number that had in fact been preexposed, and were at least potentially preparasitized, was denoted by the discrete, interval-scale variable number of hosts preexposed. As in the second study, however, interactions of a dichotomous replication vector were used to distinguish between the corresponding parameters of the constructive replications represented by Experiment 1 (vector $=0$ ) and Experiment 2 (vector $=1$ ), where these were concurrently estimated within a common model for comparison.

For Experiment 2, six additional predictor variables that were not directly manipulated were also afterwards observed and recorded. The first three extra predictors were number of hosts yielding oyster-eyed, number of hosts yielding scarlet-eyed, and number of hosts yielding diapausing jewel-wasp offspring. A pupa was determined to have yielded any given type of wasp when it was observed to produce at least one wasp offspring individual of that type. A single host could therefore be concurrently counted as yielding more than one type of wasp if it produced wasp offspring of more than one type; the different numbers of hosts so classified could therefore have overlapping domains and be summed to exceed that of the combined host sample. The wasp offspring that were either identifiably oyster-eyed, scarlet-eyed, or diapausing, however, constituted mutually exclusive categories and defined the last three of the extra predictors: number of wasp offspring oystereyed, number of wasp offspring scarlet-eyed, and number of wasp offspring diapausing.

\section{Statistical Analyses}

Because only housefly pupae were used for the adult exposures, hypotheses regarding differential suitability of alternative host species (Figueredo, 1989b) were not relevant to this study, and it was deemed unnecessary to examine the excess host mortality for disproportionate "overkill" by including the host fly survivorship criterion in the statistical analyses. Because successful parasitization of test pupae was the only dependent variable modeled, singlecriterion multiple regression techniques were applied, using the SAS (Ray, 1982) REG procedure.

Two alternative curvilinear transformations, suggested by psychophysical theory, were performed upon the predictor variables. These were the natural logarithmic and the square root, or $1 / 2$ power, transformation. Certain predictors, however, could assume a meaningful value of 0 , which has an undefined natural logarithm. Since the logarithm of 1 equals 0 , however, a constant of 1 was added to each score prior to performing that particular transformation. This standard procedure has no statistical impact other than permitting the data transformed to include the raw scale value of 0 (Cohen \& Cohen, 1983). Both the rationale and the proposed interpretations for these curvilinear functions will be discussed in the appropriate sections below.

The most parsimonious and predictive models were arrived at by evaluating a series of alternative and hierarchically nested multiple regression models. These models were separately tested for statistical significance using $F$ ratios, derived from the squared multiple correlations (SMCs), and the whole model degrees of freedom. These statistics were directly compared between alternative non-nested models. For hierarchically nested models, semipartial $F$ ratios, derived from the difference or semipartial squared multiple correlations (SSMCs) and the difference degrees of freedom were used to test the statistical significance of any regression parameters specified only in the more inclusive of the nested models (Cohen \& Cohen, 1983). This is a formal procedure for selecting the optimal regression model, ideally requiring the minimal number of parameter estimates to account for the maximal proportion of systematic variance. For logical and mathematical consistency, all the predictors in each separate multiple regression model, as well as each hierarchically nested series thereof, were subjected to the same uniform data transformation. Whole models and statistical arguments were therefore based on either rectilinear, logarithmic, or power functions, and not on any post hoc combination of transformed predictors.

\section{RESULTS}

\section{General Description}

The results obtained from any of the experimental wasps that died during either the conditioning or the testing exposure were omitted from the regression models. These numbered 20 out of 180 wasps in Experiment 1, and 28 out of 160 wasps in Experiment 2. Because of this unexplained mortality, not all of the pupae that were initially assigned for host conditioning were ultimately used in the analyses. Those preexposed to confederate wasps numbered 312 out of 360 pupae for Experiment 1, and 327 out of $\mathbf{4 4 0}$ pupae for Experiment 2. Those not preexposed to confederate wasps numbered 338 out of 360 pupae for Experiment 1, and 394 out of 440 pupae for Experiment 2.

Because all hosts in Experiment 1 produced mutually indistinguishable brown-eyed wasp offspring, it was not possible to discriminate between the conditioning pupae that had been successfully preparasitized by the confederates and those that were thereafter successfully parasitized by the experimentals. Because these offspring were genetically marked in Experiment 2, however, the following results were obtained: 186 of the conditioning pupae were successfully preparasitized by the confederates, ultimately producing 1,238 scarlet-eyed wasp offspring; 40 conditioning pupae were successfully parasitized by the experimentals, producing 191 oyster-eyed wasp offspring; 89 conditioning pupae produced 332 diapausing (persistently immature) wasp offspring of unidentified maternal genotype. As permitted in data encoding, certain individual hosts of the latter type were concurrently counted as also successfully parasitized by either of the two identifiable genotypes.

Also because of the noted wasp mortality, only 1,752 (960 in Experiment 1, and 792 in Experiment 2) out of the 2,040 pupae that were assigned for subsequent testing were exposed to the surviving experimental wasps. Of these, 1,125 (582 in Experiment 1, and 543 in Experiment 2) were successfully parasitized exclusively by the experimental wasps. In Experiment 2, these produced 3,301 oyster-eyed wasp offspring.

\section{Alternative Regressions Models}

Table 1 presents the bivariate and multiple regressions upon subsequent host acceptance of the logarithmic transformation of conditioning hosts not previously exposed to confederates (number of hosts not preexposed) that were 
Table 1

Replication, Combination and Hierarchical Regressions: Hosts Not Preexposed versus Hosts Preexposed (Logarithmic Transformations)

\begin{tabular}{lccc}
\hline & Experiment 1 & Experiment 2 & Combined \\
\hline Intercept & $0.543(0.034)$ & $0.601(0.036)$ & $0.541(0.027)$ \\
Not Preexposed* & $0.072(0.030)$ & $0.076(0.026)$ & $0.074(0.020)$ \\
Replication & & & $0.062(0.031)$ \\
SMC & 0.0358 & 0.0596 & 0.0660 \\
$F$ & 5.875 & 8.237 & 10.203 \\
$d f$ & 1,158 & 1,130 & 2,289 \\
$p\left(H_{0}\right)$ & 0.0165 & 0.0048 & 0.0001 \\
Preexposed $\dagger$ & $0.009(0.033)$ & $-0.037(0.027)$ & $-0.013(0.021)$ \\
SSMC & 0.0005 & 0.0131 & 0.0012 \\
$F$ & 0.0815 & 1.8224 & 0.3705 \\
$d f$ & 1,157 & 1,129 & 1,288 \\
$p\left(H_{0}\right)$ & 0.7757 & 0.1794 & 0.5432 \\
\hline
\end{tabular}

Note-Standard errors are given in parentheses. SMC $=$ squared multiple correlation, SSMC = semipartial squared multiple correlation. *Number of hosts not preexposed. †Number of hosts preexposed.

presented to each experimental wasp. The successive columns contain the results of Experiment 1, Experiment 2, and Experiments 1 and 2 combined. The rows contain the unstandardized intercept and regression weight estimates, with standard errors in parentheses, the whole model squared correlations (SMCs), $F$ ratios, degrees of freedom, and probabilities under the null hypothesis. For the combined sample, the parameter estimate for replication indicates a difference in intercept between experiments. This difference will be addressed further below. All models, however, were statistically significant. The combined sample regression had higher squared correlations than either single experiment, and Experiment 2 had higher squared correlations than did Experiment 1.

For both experiments, whether modeled singly or in combination, either of the curvilinear regressions, logarithmic or square root, had higher squared correlations than did the rectilinear regressions. Although the square root transformations sometimes yielded slightly higher squared correlations than did the logarithmic models, this difference was negligible. The predicted criterion scores for the range of predictor values tested were compared for the curvilinear functions and found to fall within virtual rounding error of each other. None of the regression models had very high absolute squared correlations. This is probably attributable to the restriction of range in this study relative to either of the two preceding studies (Figueredo, 1989a, 1989b), since the conditions manipulated were comparatively much fewer and more similar.

\section{Hierarchically Nested Regression Models}

Table 1 also presents the hierarchical regressions for the incremental effects upon subsequent host acceptance of the logarithmic transformation of conditioning hosts previously exposed to the confederates (number of hosts preexposed) that were presented to each experimental wasp. The extra parameter was estimated hierarchically after the logarithm of number of hosts not preexposed and the replication vector, where applicable. The successive columns again contain the results of Experiment 1, Experiment 2, and Experiments 1 and 2 combined. The rows contain the unstandardized intercept and regression weight estimates, with standard errors in parentheses, the semipartial squared multiple correlations (SSMCs), semipartial $F$ ratios, difference degrees of freedom, and associated probabilities under the null hypothesis.

Results of these analyses indicate that the incremental effects of the preexposed hosts in Experiments 1 and 2, whether modeled singly or in combination, were statistically nonsignificant and inverse in directionality between experiments. Hierarchical regressions were also used to test the incremental interaction effects of both curvilinear transformations of number of hosts not preexposed with number of hosts preexposed. These incremental interaction estimates also were statistically nonsignificant and suggestively inverse in directionality between experiments. Again, the same pattern of outcomes were obtained for the rectilinear functions, but parallel analyses are not reported due to the predictive inferiority of that model.

Most striking is the virtual reproduction of parameter estimates between the two experiments. The statistical significance of these replications was tested by hierarchical regression. Table 2 lists the results of these analyses for both the intercepts and the slopes of the logarithmic transformations. The successive columns contain the SSMCs, semipartial $F$ ratios, and the associated probabilities under the null hypothesis. Both the extra intercept and the extra slope parameters tested, the replication vector and its in-

Table 2

Hierarchical Regressions: Replication Vector and Interaction with Hosts Not Preexposed (Logarithmic Transformation)

\begin{tabular}{lcccc}
\hline & SSMC & $F$ & $d f$ & $p\left(H_{0}\right)$ \\
\hline $\begin{array}{l}\text { Replication vector } \\
\text { Replication vector }\end{array}$ & 0.0135 & 4.1772 & 1,289 & 0.0419 \\
$\times$ not preexposed* & 0.0000 & 0.0000 & 1,288 & 1.0000 \\
\hline
\end{tabular}

*Not preexposed $=$ number of hosts not preexposed. 
teraction with the logarithm of number of hosts not preexposed, were estimated hierarchically after the main effects. The SSMC was barely significant for the replication vector and was unequivocally nonsignificant for the interaction term. This indicates that, although the intercepts might have varied slightly between constructive replications, the slopes of the functions remained statistically constant. The variance between the intercepts probably reflects systematic, but otherwise inconsequential, sampling differences in mean host quality between the successive cohorts of pupae used for the two experiments. The same outcome was obtained for the rectilinear function, but parallel analyses are not reported due to the predictive inferiority of that model.

A qualitative outcome of Class 1 , as is described in the introduction, was thus unequivocally obtained and crossvalidated in two independent samples. This means that presentation of any tested number of preparasitized hosts during conditioning, whether alone or in any combination with unparasitized hosts, produced no significant effect upon subsequent host acceptance. The hypothesis that response thresholds are modulated by expected hostspecies mean profitabilities is not supported. The competing hypothesis that response thresholds are modulated by expected gross host-species abundances, however, must be qualified in that only the net abundance of unparasitized hosts apparently affects parasitoid responsiveness. Because it was suggested that this outcome might be comparable to Waage's (1979) threshold rate model of waning kairomonal responsiveness for Nemeritis canescens, wherein only the consummatory response (oviposition) rate, and not the total appetitive stimulus (encounter) rate, is utilized as a host-abundance cue, certain hypotheses derived from that analogy were subjected to more detailed scrutiny.

\section{Hypotheses Involving Additional Predictors}

Table 3 lists both the simple bivariate and the hierarchically nested effects of the logarithmic transformations of the additional predictors that were observed and recorded for Experiment 2. These analyses were therefore restricted to that data set. For the simple bivariate regressions, the successive columns contain the bivariate squared correlations (SMCs), $F$ ratios, and probabilities under the null hypothesis. For the hierarchical regressions, the successive columns contain the SSMCs, semipartial $F$ ratios, and associated probabilities under the null hypothesis. Each of the additional parameters were estimated hierarchically after the logarithm of number of hosts not preexposed.

In the bivariate regressions, only one of the predictors tested emerged as statistically significant. Although the logarithmic transformation of number of wasp offspring diapausing was found significant, the bivariate squared correlation was appreciably lower than that of number of hosts not preexposed. In the hierarchical regressions, none of the additional predictors tested emerged as statistically significant. Although the whole models were significant, the SMCs of models including both the logarithm of num-
Table 3

Bivariate and Hierarchical Regressions: Hosts Yielding Parasitoids and Wasp Offspring Produced (Logarithmic Transformations)

\begin{tabular}{lccc}
\hline & SMC & $F(1,130)$ & $p\left(H_{0}\right)$ \\
\hline $\begin{array}{l}\text { Number of hosts yielding } \\
\text { oyster-eyed }\end{array}$ & 0.0097 & 1.271 & 0.2616 \\
$\begin{array}{l}\text { scarlet-eyed } \\
\text { diapausing }\end{array}$ & 0.0066 & 0.865 & 0.3540 \\
$\begin{array}{l}\text { Number of wasp offspring: } \\
\text { oyster-eyed }\end{array}$ & 0.0281 & 3.755 & 0.0548 \\
scarlet-eyed & 0.0161 & 2.132 & \\
diapausing & 0.0138 & 1.825 & 0.1467 \\
& 0.0337 & 4.528 & 0.1790 \\
& & & \\
& SSMC & $F(1,129)$ & \\
Number of hosts yielding & & & \\
oyster-eyed & 0.0000 & 0.000 & 1.0000 \\
scarlet-eyed & 0.0069 & 0.954 & 0.3305 \\
diapausing & 0.0056 & 0.773 & 0.3809 \\
Number of wasp offspring: & & & \\
oyster-eyed & 0.0015 & 0.206 & 0.6507 \\
scarlet-eyed & 0.0123 & 1.170 & 0.1933 \\
diapausing & 0.0106 & 1.471 & 0.2274 \\
\hline
\end{tabular}

ber of hosts not preexposed and either that of number of hosts yielding oyster-eyed, number of hosts yielding scarlet-eyed, number of hosts yielding diapausing, number of wasp offspring oyster-eyed, number of wasp offspring scarlet-eyed, or number of wasp offspring diapausing were not significantly higher than that of the model with the logarithm of number of hosts not preexposed alone.

In addition, hierarchical regressions were performed in which all these additional predictors, taken together, were estimated after the logarithm of number of hosts not preexposed (first including, and then excluding, that of number of hosts preexposed) and tested "setwise" against the simpler bivariate model. These results indicated that the setwise SSMCs were nonsignificant for the total difference degrees of freedom. This confirmed the hypothesis that no statistical suppression relationship between the predictors produced their individual insignificance (Cohen $\&$ Cohen, 1983). As before, the same pattern of outcomes was obtained for the rectilinear functions, but parallel analyses are not reported due to the predictive inferiority of that model. All of this indicates that no function form of any of the additional predictors tested, whether singly or in combination, either (1) statistically equaled when modeled in substitution for, or (2) significantly exceeded when modeled in addition to, the predictive power of the logarithm of number of hosts not preexposed alone. Thus, in this study, no hypotheses related to the Waage (1979) threshold rate model were supported for this species of parasitoid.

\section{DISCUSSION}

\section{Qualitative Description}

The effect of host conditioning upon subsequent host acceptance therefore appears to be exclusively attributable to the number of unparasitized hosts encountered by 
the wasp during conditioning. This effect of only fully acceptable hosts available to the wasp is not indirectly mediated by either (1) the number of hosts successfully accepted for oviposition by that wasp or (2) the number of eggs successfully deposited in those hosts by that wasp during the conditioning exposure. Furthermore, the effect is not mitigated by either (3) the number of hosts encountered that had been exposed to other wasps, (4) the number of hosts encountered that had been successfully accepted for oviposition by other wasps, or (5) the number of eggs that had been successfully deposited in those hosts by other wasps prior to the conditioning exposure. Finally, the estimation of this effect was not qualified by either (6) the number of unassignable hosts encountered that had been successfully accepted for oviposition or (7) the number of unassignable eggs encountered that were successfully deposited by that or any other wasp.

Although one can never prove the null hypothesis, the idea that assessment of local host-species abundances in this parasitoid is mediated by some crude physiological mechanism that simply registers oviposition rates, rather than the processing and integration of foraging information available from multiple host-species cues (see Taylor, 1974), would seem to be falsified by the nonsignificance of Factors 1 and 2 above. Similarly qualified, the apparently reasonable prediction that a gross numerical practice effect of host experience can function to decrease future searching or handling times, and so enhance attack rates, would seem to be seriously compromised by nonsignificance of Factors 3 and 4.

These results also contradict the notion that the effects of host conditioning in this parasitoid merely reflect the trained enhancement of motoric performance with repeated execution of a stereotyped response. On the other hand, adaptive modulation of kairomonal "responsiveness thresholds" would seem to require only an alteration in the host-cue response bias, whereas more apparently cognitive alternatives, such as "search image formation" (sensu Tinbergen, 1960), minimally require a measurable increase in host-cue discriminability. Although the foraging behavior of the jewel wasp did not lend itself readily to the formal techniques of the theory of signal detection, the controlling factors that have been identified as driving that model were found amenable to experimental manipulation and the predicted outcomes to statistical measurement.

Therefore, of the two nonperceptual factors documented in the psychophysical literature to account for labile response criteria, the a posteriori subjective probability of stimulus presentation, and not the expected payoff matrix of observer decision outcomes, was supported as mediating jewel-wasp host conditioning. Translated into corresponding jargon for behavioral ecology, the parasitoid's expected encounter rate with unparasitized host individuals, based upon prior host-species experience, and not the expected profitability of that host species, as modified by any changes in parasitoid searching or handling times produced by prior host-species experience, modu- lates the subsequent attack rate for that host species. For this translation, however, the probability of stimulus presentation must be narrowly defined as reflecting the net local abundance of fresh host individuals and not the gross local abundance of the host species, regardless of prior attack by conspecifics. These encounter rates are "computed" during adult host conditioning only for previously unparasitized pupae of each species, stochastically indicating only the abundance of fresh and fully acceptable individuals of that type, "discounted" for the local competition for that particular host population. The expressed effects of these experiential summations upon the separate host-species attack rates are curvilinear and convex, but the conventional wisdom in theoretical ecology is that this merely reflects the relative inflexibility of performance constraints (such as minimum handling time).

As qualified above, therefore, single-species host conditioning operates by changing the expected host encounter rate, or subjective stimulus presentation probability, for that species. It does not operate by altering the relative profitabilities of alternative host species or the payoff matrix of observer decision outcomes. These psychophysical host-species cues presumably either include or merely represent the kairomonal semiochemicals described above. Because of the apparent productivity of the general analogy with the cited kairomonal responsiveness model, certain specific hypotheses were tested that pursue the analogy further. However, none of these additional hypotheses were supported by the data, suggesting that, although the final output characteristics (of adaptively modulated kairomonal responsiveness thresholds) may be similar between these models of parasitoid behavior, the initial input and integrative characteristics (of host-cue information acquisition and processing) appear to be very dissimilar. The details of the latter were not fully revealed for this parasitoid by the present investigation, but mediating mechanisms roughly equivalent to the cited threshold rate model were clearly not supportable.

\section{Function Form Interpretations}

Both of the best-fitting function forms, the natural logarithmic and the square root transformation, reported for the effects of host conditioning upon subsequent host acceptance were curvilinear and negatively accelerated. Psychophysically, the resemblance was suggested (Perrott, personal communication, 1987) to the classical function forms of the Fechner-Weber and the Stevens Power law. Although no statistical basis was found for selecting one of these transformations over the other, this situation was viewed as reflecting the current state of the controversy regarding the "correct" form of the psychophysical function. This comparison was intended as merely suggestive, rather than conclusive, of curvilinear host-conditioning functions reflecting a perceptual, rather than a performance, constraint.

Ecologically, these curvilinear functions superficially resemble Holling's (1959) Type II, or convex, functional response. This was, indeed, the type of functional response 
previously reported for this parasitoid (DeBach \& Smith, 1941). The abscissa for the functions reported here, however, represent prior host conditioning rather than current host population density. Such an increase in attack rate with host experience is predicted to produce a Type III, or sigmoid, functional response, which is consistent with the theoretical expectation for a "switching" parasitoid (Hassell, 1978). Apparently, DeBach and Smith (1941) used wasps that were reared on and then exposed for adult testing to the same host species. Thus, it is conceivable that the results obtained for the host densities experienced during parasitoid adulthood reflect the shape of the functional response already brought past the inflection point by preimaginal conditioning. The present study used wasps that were reared on a host species different from that used for the adult conditioning and testing exposures. This procedure permitted the monitoring of the initial portion of the parasitoid functional response to perceived host density from a more behaviorally valid host experience of one. This comparison, as before, is intended as speculative rather than conclusive, however, and any contradictions between studies could be attributable to the other major differences in design.

\section{Summary}

The following results were obtained and cross-validated in two independent samples:

1. The hypothesis that response thresholds are modulated by expected host-species abundances was supported, but qualified in that only the expected net abundances of unparasitized host-species individuals were critical. Both best-fitting function forms reported for this effect were curvilinear and convex.

2. The competing hypothesis that response thresholds are modulated by expected host-species mean profitabilities was not supported. The presentation of previously parasitized hosts during conditioning produced no significant effect upon subsequent host acceptance. This simultaneously contradicted both the host-species "practice effect" and "tracking of quality" hypotheses.

3. Hypotheses derived from analogy with the Waage threshold rate model of optimal kairomonal responsiveness were not supported. No transformation of any additional predictors suggested by that analogy, whether singly or in combination, either statistically equaled when modeled in substitution for, or significantly exceeded when modeled in addition to the predictive power of either transformation of the absolute number of unparasitized hosts encountered.

\section{REFERENCES}

Cock, M. J. W. (1978). The assessment of preference. Journal of Animal Ecology, 47, 805-816.

COHEN, J., \& COHEN, P. (1983). Applied multiple regression/correlation analysis for the behavioral sciences (2nd ed.). Hillsdale, NJ: Erlbaum.

Cornell, H. V., \& Pimentel, D. (1978). Switching in the parasitoid Nasonia vitripennis and its effects upon host competition. Ecology, 59, 297-308.
Craighead, F. C. (1921). Hopkins host-selection principle as related to certain cerambycid beetles. Joumal of Agricultural Research (U.S.), 22, 189-220.

DeBACH, P., \& SMITh, H. S. (1941). The effect of host density on the rate of reproduction of entomophagous parasites. Journal of Economic Entomology, 34, 741-745.

Dember, W. N., \& WarM, J. S. (1979). Psychology of perception (2nd ed.). New York: Holt, Rinehart \& Winston.

EDWARDS, R. L. (1954). The host-finding and oviposition behavior of Mormoniella vitripennis (Walker), a parasite of muscoid flies. Behavior, 7, 88-112.

FIGUEREDO, A. J. (1987). The statistical measurement, developmental mechanisms, and adaptive ecological functions of conditioned hostselection in the parasitoid jewel wasp. Unpublished doctoral dissertation, University of California, Riverside.

FIGUEREDO, A. J. (1989a). Behavioral interactions of adult foraging experience with preimaginal conditioning in the jewel wasp. Manuscript submitted for publication.

FIGUEREDO, A. J. (1989b). Path analysis of preimaginal conditioning in the jewel wasp. Manuscript submitted for publication.

HASSELL, M. P. (1978). The dynamics of arthropod predator-prey systems. Princeton: Princeton University Press.

Holling, C. S. (1959). The components of predation as revealed by a study of small mammal predation of the European pine sawfly. Canadian Entomologist, 91, 293-320.

HopkINs, A. D. (1917). Discussion of presidential address. Journal of Economic Entomology, 10, 92-93.

KLING, J. W., \& RigGs, L. A. (1971). Woodworth \& Schlosberg's experimental psychology (3rd ed.). New York: Holt, Rinehart \& Winston.

KreBS, J. R., \& MCCleery, R. H. (1984). Optimization in behavioral ecology. In J. R. Krebs \& N. B. Davies (Eds.), Behavioral ecology: An evolutionary approach (2nd ed., pp. 91-121). Sunderland, MA: Sinauer.

Miller, J. R., \& Strickler, K. L. (1984). Finding and accepting host plants. In W. J. Bell \& R. T. Carde (Eds.), Chemical ecology of insects (pp. 127-157). Sunderland, MA: Sinauer

MURDOCH, W. W. (1969). Switching in general predators: Experiments on predator specificity and the stability of prey populations. Ecological Monographs, 39, 335-354.

Nordlund, D. A. (1981). Semiochemicals: A review of the terminology. In D. A. Nordlund, R. L. Jones, \& W. J. Lewis (Eds.), Semiochemicals: Their role in pest control (pp. 13-28). New York: Wiley.

RAY, A. A. (1982). SAS user's guide: Statistics. Cary, NC: SAS Institute.

SMith, M. A., \& CoRNell, H. V. (1979). Hopkins host-selection in Nasonia vitripennis and its implications for sympatric speciation. Animal Behaviour, 27, 365-370.

TAYLOR, R. J. (1974). Role of learning in insect parasitism. Ecological Monographs, 44, 89-104.

THORPE, W. H. (1938). Further experiments on olfactory conditioning in a parasitic insect. The nature of the conditioning process. Proceedings of the Royal Society of London, B126, 370-397.

THORPE, W. H. (1939). Further studies on preimaginal olfactory conditioning in insects. Proceedings of the Royal Society of London, B127, 424-433.

THORPE, W. H., \& JONES, F. G. W. (1937). Olfactory conditioning in a parasitic insect and its relation to the problem of host selection. Proceedings of the Royal Society of London, B124, 56-81.

Tinbergen, L. (1960). The natural control of insects in pinewoods, 1: Factors influencing the intensity of predation by songbirds. Archives Neerlandaises de Zoologie, 13, 266-336.

Vinson, S. B. (1984). Parasitoid-host relationship. In W. J. Bell \& R. T. Carde (Eds.), Chemical ecology of insects (pp. 205-233). Sunderland, MA: Sinauer.

WAAGE, J. K. (1979). Foraging for patchily-distributed hosts by a parasitoid, Nemeritis canescens. Joumal of Animal Ecology, 48, 353-371.

WALKER, I. (1967). Effect of population density on the viability and fecundity in Nasonia vitripennis Walker (Hymenoptera: Pteromalidae.). Ecology, 48, 294-301.

Whiting, A. (1967). Biology of Mormoniella. Quarterly Review of Biology, 42, 334-406. 
WyLie, H. G. (1958). Factors that affect host finding by Nasonia vitripennis (Walk.) (Hymenoptera: Pteromalidae.). Canadian Entomologist, 90, 597-608.

WyLIE, H. G. (1965). Discrimination between parasitized and unparasitized house fly pupae by females of Nasonia vitripennis (Walk.) (Hymenoptera: Pteromalidae.). Canadian Entomologist, 97, 279-286.

\section{APPENDIX}

\section{The Natural History of the Jewel Wasp}

The jewel wasp, Nasonia (Mormoniella) vitripennis (Hymenoptera: Pteromalidae), is an oligophagous, gregarious ectoparasitoid of the pupae of an estimated 68 species of muscoid flies (Whiting, 1967). The term oligophagous, which denotes feeding upon a few host species, refers to an intermediate level of ecological specialization between monophagous, which denotes feeding exclusively upon a single host species, and polyphagous, which denotes feeding facultatively upon a wide variety of host species. The term parasitoid refers to an intermediate category of ecological relationship, between the extremes of predator, which first kills and then consumes possibly multiple prey individuals, and that of true parasite, which slowly feeds upon but presumably never kills what is often a single host individual. A parasitoid denotes an ecological class of consumer that may first slowly feed upon but ultimately kills its single host individual. The qualifiers, ecto- and gregarious, indicate that parasitoid development occurs outside the living tissues of the host and that multiple parasitoids may commonly occur upon a single host individual.

After emergence from the host and subsequent copulation (not always required, due to haplodiploidy), a female jewel wasp will seek out the pupal stage of any of its host fly species. The location by the female of a host pupa will then release a relatively invariant sequence of behavioral responses (Edwards, 1954). These are touching (initial contact), drumming (with antennae), tapping (with tip of abdomen), drilling (with ovipositor), egglaying (if host is accepted), withdrawal (of ovipositor), and, finally, feeding (upon host body fluids). The eggs are laid within the space inside the puparium wall of the host insect, but outside the living body of the fly pupa. The juvenile parasitoids develop there completely to maturity, progressively feeding upon and ultimately devouring most or all of the host tissues. A jewel wasp typically spends about 2 days as an egg, 6 days as a larva, 6 days as a pupa, and about 30 days (if male) to 45 days (if female) thereafter as an adult.

Many of the fly species utilized as hosts by the jewel wasp, including both of those used in these studies, themselves feed upon and consequently constitute ephemeral food sources (e.g., carrion, dung, and other organic detritus). They are classical ecologically "opportunistic" or "fugitive"' species, specializing in the rapid colonization of new microhabitats and suffering frequent local extinctions. Proverbially, they breed like flies, and they die like flies. For such species, one would therefore expect the local abundances to be subject to relative instability and local distributions to considerable unpredictability in time and space. The first, and perhaps the most challenging, stage in the exploitation of such species as prey is therefore the location of suitable-host habitat (Vinson, 1984). There is some evidence that this behavioral stage is mediated by specific airborne olfactory cues known as kairomones in the field of chemical ecology. Contact and olfactory chemoreception are also almost certainly involved in the subsequent stages of individual host finding and that of host acceptance. Once these premium host microhabitats have been located, however, the optimal exploi- tation of host species found there generates its own suite of selective pressures. It is with the evolutionary products of those behavioral pressures that this study is concerned.

\section{Developmentally Conditioned Host Acceptance}

The facilitation of oviposition by an adult insect upon the host species on which it was reared as a larva is called Hopkins host selection (Hopkins, 1917; see also Craighead, 1921) or preimaginal conditioning (Thorpe, 1938, 1939; Thorpe \& Jones 1937). The strategy of disproportionate predation by any predator or parasite upon the prey or host species that is locally encountered in greatest abundance is called switching (Hassell, 1978; Murdoch, 1969). In the jewel wasp, both Hopkins host selection (Smith \& Cornell, 1979) and adult switching between host species (Cornell \& Pimentel, 1978) have been separately reported. Both of these reports describe the effect of jewel-wasp host conditioning as a "shift in host preference" rather than a reversal of such preference in the direction of the host species experienced at either the larval or the adult stage of development.

Although the concurrent existence of both Hopkins host selection and adult switching between host species in the jewel wasp was confirmed in subsequent investigations (Figueredo, 1989a, $1989 \mathrm{~b}$ ), the main effects observed of either preimaginal feeding or adult foraging experience with either species of host were to increase the acceptance by the wasp of that particular host species, regardless of the foraging alternatives present. These subsequent studies were not temporally extended enough to determine whether any eventual forgetting of prior experience would occur, but neither proactive nor retroactive inhibition of host-species conditioning was shown to occur. The magnitudes of the effects of adult foraging experience were found to be statistically unaffected by the host species of preimaginal conditioning. Successively acquired increments in acceptance for different host species were thus successfully modeled as being simply additive, neither precluding nor superceding each other across development.

No qualitative difference was found between the effects of preimaginal conditioning and those of subsequent adult foraging experience. Furthermore, preimaginal conditioning was shown to have persistent behavioral effects in the face of what has been called "switching" during adulthood. Adult foraging experience was shown to complement, rather than to abolish, early experience in a cumulative conditioning process. Hopkins host selection and switching can therefore be interpreted as merely developmentally dichotomized manifestations of a common learning mechanism that subserve a common ecological function.

\section{Persistent Host Preference}

The existence of a true host-species "preference" in the jewel wasp, however, was also confirmed in the recent studies (Figueredo, 1989a, 1989b). Host preference had also been reported by the prior researchers, but it had not been unequivocally demonstrated. For assessing preference, alternative host species had merely been provided to the wasps in equal numbers without any consideration of whether their simultaneous presence, when so paired, altered the host-acceptance patterns for either or both of the given alternatives (Cock, 1978; Miller \& Strickler, 1984). The subsequent research was designed to compare the results of such numerically balanced betweenspecies choices with those of independently conducted withinspecies choices of alternative hosts. The separate baseline levels of acceptance for each host species were thus measured when 
no hosts of alternative species were simultaneously present. This procedure confirmed that the contiguity of foraging alternatives indeed affected the immediate host-acceptance decisions of this parasitoid.

The preference so indicated was for blowfly over housefly pupae. Preimaginal conditioning, even when followed by adult experience with the same host species, was not shown to alter this behavioral dimension. Only mixed-species experience across developmental stages, preimaginal conditioning with either alternative host species followed by adult exposure to the other, was found to affect the magnitude of host preference. This effect, however, was to further enhance, rather than diminish, the preference for blowfly hosts, regardless of the order in which the two alternative species were successively experienced during development. This finding is in marked contrast with those reported by prior researchers, who separately reported quantitative shifts in the magnitude of host-species preference with both Hopkins host selection and switching, sometimes away from the preferred host species, in spite of the qualitative invariance in the direction of host preference.

\section{Optimal Foraging Information}

The ultimate ecological function of conditioned host selection in the jewel wasp was strongly suggested by the results of these studies. This adaptive microevolutionary explanation involves the initial assessment and continuous tracking by the parasitoid of the separate and current abundances of the alternative host species in its local environment. This is accomplished by the detection, decoding, and integration of various hostspecies cues available to the parasitoid throughout development. The exact proximate mediation of this host-cue information processing will be considered in greater mechanistic detail below. For the present, however, the normative, rationalistic, and narrative mode of optimal foraging theory will be employed to expound on the proposed selective advantage of the behavioral mechanism.

The first indication that the newly emerged adult parasitoid receives regarding local foraging conditions is the host species of its larval rearing. Unfortunately, this information may be somewhat obsolete, reflecting the conditions experienced by the prior parental generation. Nevertheless, if a parasitoid detects preimaginally that it was deposited by its mother in a less preferred host-species individual, this datum may stochastically indicate that any more-preferred host species is either locally or currently unavailable. Any less preferred host-species individuals encountered should therefore not be unduly rejected by the newly emerged parasitoid until this dire inference has been refuted by more current information. On the other hand, if the parasitoid detects preimaginally that it was deposited by its mother in a more preferred host-species individual, this datum may stochastically indicate that any more-preferred host species might indeed be both locally and currently available. Any lesspreferred host-species individuals encountered should therefore be confidently rejected by the parasitoid until this hopeful inference has been sufficiently refuted by more current information.

The tracking of host-species abundances, therefore, continues during adulthood. As host pupae are encountered by the parasitoid during adult foraging, the acceptance thresholds for different host species are separately and commensurately modulated by the corresponding host encounter rates. As described above, host-species preference in the jewel wasp is apparently innate and irreversible, even in gross magnitude. The selective advantage of this is obvious from the results of these studies, since at least one of the two determining factors have been separately examined in detail. Ovipositing in the preferred host species increases both the quantity (per host pupa) and quality (future female fecundity) of the offspring produced (Figueredo, 1987). Provided that differential host-species experience does not alter the relative searching or handling times between alternative host species, these results suggest that the profitabilities of the alternative host species are unequal and fixed. Roughly speaking, profitabilities can be considered proportional to the ratios between these opposing factors, balancing the benefits in gross food value gained against the opportunity costs in time invested. Because, according to optimal foraging theory, the rank order of host-species preference should reflect that of the host-species profitabilities, fixed profitabilities should naturally select for fixed preferences. This interpretation is not necessarily contradicted by the reported developmental increase in blowfly host preference with mixed-species experience, since it is recognized in behavioral ecology that errors in discrimination can limit the expression of host preferences (Krebs \& McCleery, 1984). Reduction in such errors by gains in perceptual ability can therefore enhance an innate host preference.

According to the psychophysical interpretation offered for the proximate mediation of these effects, mixed-species host conditioning across developmental stages increases the discriminability of host cues between the alternative species, in addition to accruing the additive effects of successive single-species host conditioning for each; single-species host conditioning, whether acquired preimaginally or during adult foraging, modulates the independent response biases to the host cues for each species.

\section{Proximate Mediation Mechanisms}

Foraging in the jewel wasp has been shown by prior researchers to involve a kairomone (see Nordlund, 1981), which is a natural metabolic product of the interaction between the host species and host diet, such as excretory or eliminative (fecal) material (Edwards, 1954; Wylie, 1958). If alternative host species produce sufficiently distinct kairomones, or chemical variants thereof, either through differences in host-species metabolism or host-species diet, differential responsiveness to these host-species cues could provide a physiological basis for the independently labile host-species response thresholds reported.

Such a mechanism superficially resembles the Waage (1979) threshold rate model of waning kairomonal responsiveness for optimal foraging by an ichneumonid parasitoid. According to this model, the time rate of oviposition most recently experienced, reflecting the local acceptable-host encounter rate (patch quality), modulates the time course of spontaneous decay for parasitoid responsiveness (waning edge response) to a contact arrestant host chemical. Although the latter mechanism does not control foraging decisions between different host species, it illustrates how the mere adaptive modulation of response thresholds to prespecified (i.e., nonarbitrary), naturally host-associated chemical cues might achieve a comparable degree of flexibility in behavioral performance.

(Manuscript received December 30, 1988; revision accepted for publication September 15, 1989.) 